\title{
The effects measurement of hand massage by the auto- nomic activity and psychological indicators
}

\author{
Hiroko Kunikata', Kumi Watanabe ${ }^{2}$, Makoto Miyoshi', and Tetsuya Tanioka ${ }^{4}$ \\ 1)Department of Nursing, Faculty of Health Sciences, Kagawa Prefectural University of Health Sci- \\ ences, Kagawa, Japan ; Department of Nursing, Faculty of Health and Welfare Sciences, Okayama \\ Prefectural University, Okayama, Japan ; ${ }^{3}$ Department of Medical Technology, Faculty of Health Sci- \\ ences, Kagawa Prefectural University of Health Sciences, Kagawa, Japan ; and ${ }^{4}$ Department of Nursing, \\ Institute of Health Biosciences, the University of Tokushima Graduate School, Tokushima, Japan
}

\begin{abstract}
This study examined the effects of hand massage on autonomic activity, anxiety, relaxation and sense of affinity by performing it to healthy people before applying the technic in actual clinical practice. Findings were showed below : 1) the significant increase in the pNN50 and the significant decrease in the heart rate meant the intervention of massage increased the autonomic nervous activity, improved the parasympathetic nerve activity and reduced the sympathetic nerve activity. This means the subjects were considered to be in a state of relaxation. 2) Salivary $\alpha$ amylase has been reported as a possible indicator for sympathetic nerve activity. In this study, there was no significant difference in the salivary $\alpha$ amylase despite a decrease after massage. 3) State anxiety score is temporal situational reactions while being in the state of anxiety and this score decreased significantly after massage. 4) The level of willingness to communicate with other person and the sense of affinity toward the massage-performer had a positive change of 70 percent. From this, it can be considered that a comfortable physical contact between a patient and a nursing profession, who are in a supported-supportive relationship, leads to an effect of shortening the gap in their psychological distance. J. Med. Invest. 59 : 206212, February, 2012
\end{abstract}

Keywords : hand massage, nursing skill, autonomic activity, anxiety, relaxation, sense of affinity

\section{INTRODUCTION}

The use of touch and massage is an inherent component of the nursing care. For example, Henderson $\mathrm{V}$ (1) pointed out that the value of body touch between a nurse and a patient should not be underestimated, especially where its effect is comfortable. However, as efficiency or evidence is prioritized in today's medical care, nurses having few chances

Received for publication November 22, 2011 ; accepted January $5,2012$.

Address correspondence and reprint requests to Hiroko Kunikata, Department of Nursing, Faculty of Health Sciences, Kagawa Prefectural University of Health Sciences, 281-1, Hara, Mure-cho, Takamatsu City, Kagawa 761-0123, Japan and Fax : +81-87-8701243. to touch or massage patients. Consequently, it has not been an easy situation to recognize the value of touch care in Japan (2). On the other hand, complementary and alternative medicines have been recognized together with the mainstream treatment in United States of America from 1960s. Systematic actions for complementary and alternative medicines also began in Japan during 1990s. Thereafter, nursing therapeutic methods have been developed and tested from the aspect of complementary and alternative medicine.

Hand massage is simple and convenient as it can be performed to various kinds of people in a relaxed posture without putting on or off clothing. Especially for people with mental disorders who have 
undergone the stress associated with their various psychiatric symptoms and have difficulty in maintaining their personal relationships, hand massage can be useful in reducing their pain as it is considered to have the effects of relaxation and reducing anxiety.

Regarding the effects of hand massage, common effects have been recognized by subjective evaluations. For example, the subjective feeling of relaxation was significantly increased (3-5), negative mood was significantly improved $(3,5,6)$, and anxiety level was significantly decreased after hand massage (7-9). Degirmen et al. (10) and Wang et al. (11) concluded that foot and hand massage can reduce postoperative pain in patients as an effective nursing intervention in postoperative pain control. Also, from physiological viewpoint, Sato et al. (3) reported that the heart rate and the $\mathrm{LF} / \mathrm{HF}$ ratio significantly decreased after hand massage. Many reports pointed out the heart rate were significantly decreased after hand massage $(4,10,11)$.

On the other hand, there are some researchers mentioned that the heart rate was no significantly decreased after hand massage $(5,6)$. Thus, the examination on the effects of hand massage from the aspect of the autonomic nervous activities has not necessarily established a common understanding.

For decreasing the stress of people with mental disorders whose anxiety is particularly strong, we have devised a type of hand massage based on short and soft touch. The purpose of this study is to clarify the effects of hand massage on autonomic activity, anxiety, relaxation and sense of affinity by performing it to healthy people before applying the technic in actual clinical practice.

We defined hand massage as follows ; hand massage is a nursing skill (with soft touch) conducted with the intention to make the subject feel needed.

\section{METHOD}

\section{Subjects}

The subjects were 14 healthy women student volunteers. The mean age of the subjects were $19.5 \pm$ 0.7 (range $=18-20$ ). The requirement for becoming a subject was being a woman who did not have any knowledge and skill of hand massage. This was because there was a possibility that the subjects would have positively evaluated the effects of hand massage if they had the knowledge and skills. The data were collected between February from 19 to
24, 2010.

\section{Study procedures}

\section{1) Conditions of experiment}

The experiment environment was shut off from the comings and goings of people and the outside sound. The measurement of before and after experiment were conducted in Room A. Hand massage was conducted in Room B. The temperature was 18 to 22 degrees Celsius, and the humidity level was 43 to $58 \%$ in the rooms. Sunlight was admitted into Room B. The experiment was carried from 10 A.M to the noon. The subjects were instructed to have a normal breakfast.

\section{2) Procedures}

1) After the subjects entered the room $A$ at the arranged time, they had a five minute rest on their seats with their eyes closed. 2) First, psychological indicators of anxiety and relaxation levels were measured before the hand massage. Second, the saliva of the subjects was gathered after they chewed cotton for two minutes. Then, they were asked to wear a sensor of the Pulse Analyzer Plus, and a five minute measurement was conducted after two deep breathings. While this measurement was being taken place, they were asked to keep silence. This measurement was conducted by researcher A. 3) Researcher B conducted hand massage for twenty minutes in a seated posture in Room B. 4) For the data after the massage, researcher A conducted a measurement in the room A by using the sensor of the Pulse Analyzer Plus, collected the subjects' saliva. Also, their anxiety and relaxation levels were measured using the State and Trait Anxiety Inventory (STAI) (12) and the Visual Analogue Scale (VAS).

\section{3) Method of hand massage}

The procedure of hand massage was originally prepared, consisting of the application of light finger pressure for 20 minutes. In making this original method, we referred to the therapeutic care guide made by British Red Cross Society.

1) Materials and advance preparation

Two cushions, a bath towel, a hand cream which was not to affect the autonomic nervous activities of the subjects, were prepared. The hands of researcher B was warmed up before massage.

2) Procedure and posture at hand massage

The subjects were asked to sit deeply and comfortably on a chair with a cushion on the back. Researcher B sat in front of one subject face-to-face with his knees almost touching the subject's knees. 
Two cushions were placed on the knees of both researcher B and the subject, and also a bath towel was placed on the two cushions (Figure 1).

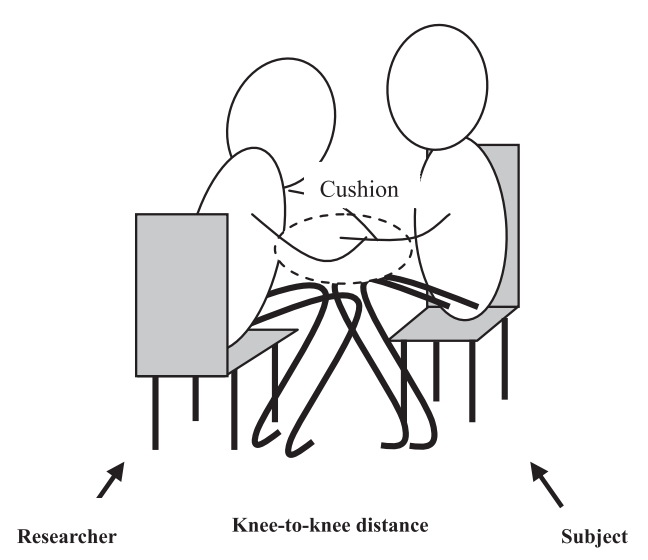

Fig. 1 Posture and position

Researcher B performed massage on the subject's fingers, dorsal surface of the hand, palms and elbows, covering the subject' body parts with both of his hands and moving from the edge toward the center of the parts. In doing so, she used her palms, elbows and shoulders smoothly in a rhythmic pace. This massage was conducted on each of the subject's hands for three minutes.

Secondly, researcher B did massage on the carpal region of the subject's hand, moving from the edge to the center just like making circles, while holding the subject's palm with one of her hands. This was done on each of the subject's hands for one minute each, and the same was also done on the dorsal surface of the subject's hands. Then, researcher $\mathrm{B}$ used her ring fingers and little fingers to hold the subject's thumb and little finger and opened her palm wide rhythmically for two minutes, and then the same was done on the other hand. Moreover, Researcher B used her balls of the fingers to do massage from the bases of the five fingers of the subject toward the tips like making circles rhythmically, while holding the subject's palm. In doing this, a little pressure was applied on the nails of the subject. This was done on both of the subject's hands for two minutes each. Lastly, researcher B placed her palm on the subject's palm and slid her gradually to the tips with her thumb clipping the subject's hand. This was done for one minute on each of the subject's hands.

4) Measure scales and instrument

After a five minute rest before measurement, the following items were used and measured : 1 )
STAI as anxiety level ; 2) salivary $\alpha$ amylase (using Benchmark Microplate Reader by Bio-Rad Laboratories and Alpha-amylase Assay Kit for saliva), and 3) finger tip pulse (using Pulse Analyzer Plus NEW TAS9 by YKC Corporation) as autonomic nervous activities. For measuring finger tip pulse, YKC Corporation's HRV (the autonomic nerve balance) analysis program was used. Heart rate was measured by time-line analysis, and frequency analysis was conducted on it. A frequency level from 0.04 to $0.15 \mathrm{~Hz}$ was classified as Low Frequency (LF), and a frequency level from 0.15 to $0.40 \mathrm{~Hz}$ was classified as High Frequency (HF). HF was considered as an indicator for parasympathetic nerve activity. The ratio between $\mathrm{LF}$ and $\mathrm{HF}$ (LF/HF) showed the overall balance of sympathetic and/or parasympathetic nerve ; it is proportional to the degree of sympathetic nerve activity and inversely proportional to parasympathetic nerve activity. pNN50 which is figured out from heart rate shows the percentage of adjacent R-R intervals which are greater than 50 ms. Thus, $50 \mathrm{~ms}$ or more are considered as an increased autonomic nervous activity.

After hand massage, in addition, changes in the conversation need level, and the senses of affinity were measured. Relaxation level was measured by using 0 to $100 \mathrm{~mm}$ Visual Analogue Scale (VAS). Changes in the level of willingness to communicate with other person and the sense of affinity were measured by setting the levels before the hand massage at the value of 0 and using VAS of -50 to 50 $\mathrm{mm}$.

\section{Study design and statistical Analysis}

This study used a before-and-after evaluation single group design. The autonomic nervous activities and psychological indicators were examined using Wilcoxon rank sum test. The HF gained from HRV was treated as a parasympathetic nerve activity indicator, LF/HF was as a sympathetic nerve activity indicator, pNN50 and salivary $\alpha$ amylase was as autonomic nerve activity indicators. Changes in the willingness to communicate with other person and the level of the sense of affinity toward Researcher $B$ were analyzed by referring to the degrees of change from the levels before massage. The analyses were performed with a commercially available statistical package, IBM SPSS statistics version 19.0 for Windows.

\section{Ethical approval of the study}

The execution of this study was approved by the 
ethical review committee of the university, to which the researchers belong. In recruiting participants, potential individuals were given an oral and written explanation about the purpose of the study, the research methods, and the advantages and disadvantages in participating. They consented to the participation after being notified that their participation was voluntary, that they could decrease their participation even in the middle of the research without any disadvantage, and that their privacy was to be protected.

\section{RESULTS}

\section{Autonomic nervous activity}

\section{1) Changes in heart rate, pNN50, $\mathrm{HF}$ and $\mathrm{LF} / \mathrm{HF}$}

The median of the heart rates before massage was 74.00 and 67.50 after massage. The heart rates after massage was significantly lower $(p<0.01)$. The median of pNN50 before massage was 72.50, and 79.50 after massage, the pNN50 after massage was significantly higher $(\mathrm{p}<0.01)$ (Figure 2$)$. The median of HF was 49.13 before and 52.38 after massage, it was not significant difference. The median of LF/HF was 1.01 before, and 0.98 after massage, it was no significant difference despite decrease (Figure 3).

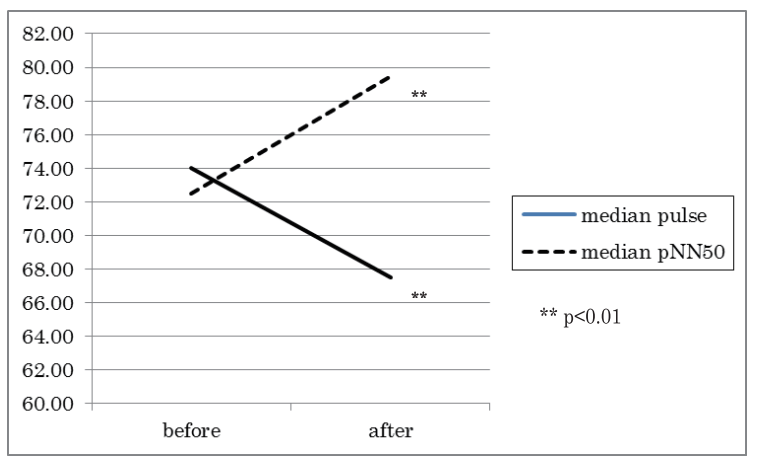

Fig. 2 Changes in heart rate and pNN50

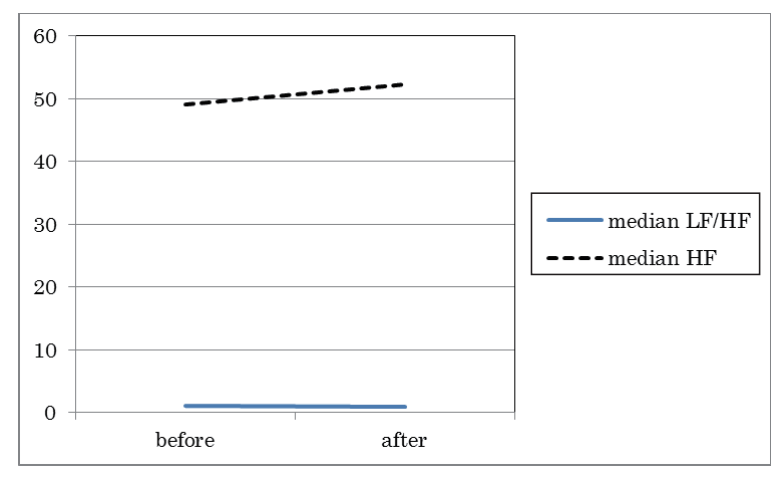

Fig. 3 Changes in HF and LF/HF

\section{2) Changes in salivary alpha amylase}

The median of salivary $\alpha$ amylase was 109.23 $\mathrm{U} / \mathrm{mL}$ before massage (range : -30.67 to 297.17 ) and $72.08 \mathrm{U} / \mathrm{mL}$ after massage (range : -61.99 to 228.62 ), it was no significant difference despite decrease after massage.

\section{The psychological indicators}

\section{1) Changes in anxiety state}

The median of state anxiety score before massage was 41.50 and 30.00 after massage which was significantly lower $(\mathrm{p}<0.01)$. The median of trait anxiety score before massage was 47.00 , and 43.00 after massage, it was significant decrease after massage $(\mathrm{p}<0.01)$ (Figure 4).

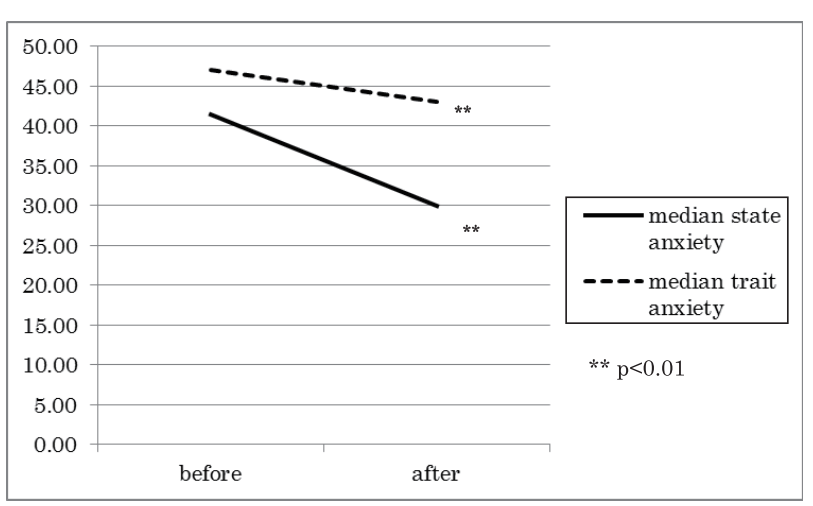

Fig. 4 Changes in state anxiety score and trait anxiety score

\section{2) Change in relaxation level}

The median of relaxation level was $42.50 \mathrm{~mm}$ before massage, and $93.50 \mathrm{~mm}$ after massage, it was significant increase after massage $(p<0.01)$ (Figure $5)$.

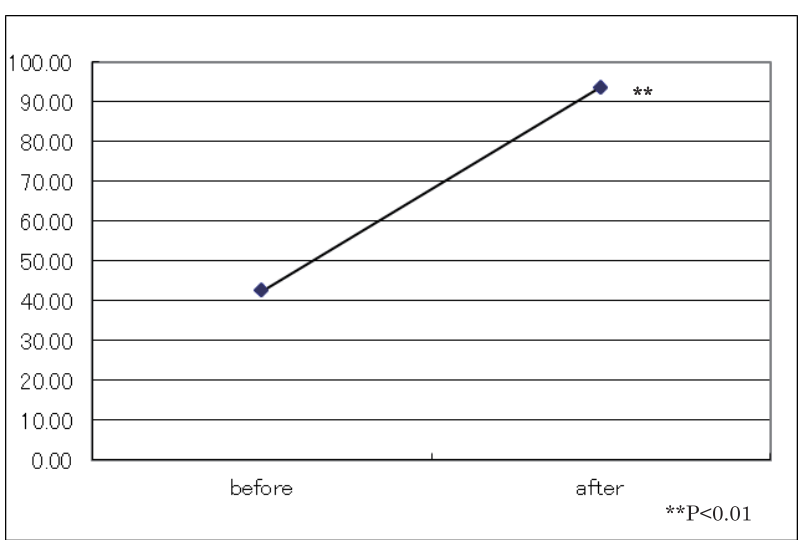

Fig. 5 Changes in relaxation level 
3) Changes in the conversation need level and the level of the sense of affinity

The median of the conversation need level was $35.00 \mathrm{~mm}$ (range : 1 to $50 \mathrm{~mm}$ ), having a positive change of 70 percent after hand massage. The median of the level of the sense of affinity was 38.50 $\mathrm{mm}$ (range : 5 to $50 \mathrm{~mm}$ ), having a positive change of 77 percent after massage (Figure 6).

Median of conversation need level: $35.00 \mathrm{~mm}$ (range 1-50mm)

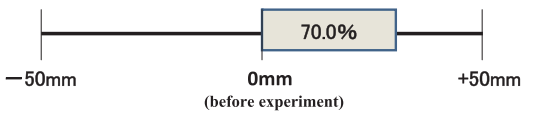

Median of sense of affinity: $38.50 \mathrm{~mm}$ (range $5-50 \mathrm{~mm}$ )

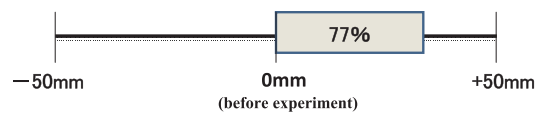

Fig. 6 Changes in conversation need level and sense of affinity

\section{DISCUSSION}

\section{The influence to the autonomic nervous activity}

Sato (3) reported that the heart rate was significantly decreased after hand massage by case-control study. In this study, a similar result was obtained that there was a significant difference after hand massage, although no comparison with a control group was made. HF is an indicator for parasympathetic nerve activity, and a high HF means a relaxed state. In this present study, the HF data was not showed significant difference after massage despite an increase in the data.

$\mathrm{LF} / \mathrm{HF}$ is proportionate to sympathetic nerve activity and inversely proportionate to parasympathetic nerve activity. Despite some previous studies recognizing significant decrease in LF/HF after massage (3), in this study was not observed significant decrease. The pNN50 which implying an increase in the autonomic nervous activity was significantly increased after massage. There was no statisticallysignificant difference. Although the group only consisted of 14 subjects, increased parasympathetic nerve was observed in 9 out of 14 subjects. The efficacy seems to vary with each person. Therefore, was considered essential that further examination for large study population applicable to investigation.

As a new indicator for the sympathetic nervous system, salivary $\alpha$ amylase has been highlighted.
Salivary $\alpha$ amylase is under the control of the sympathetic nervous system-the sympathoadrenal medullary system. At the same time, there exists a controlling system activated by direct nerve actions (13, $14)$. For this reason, salivary $\alpha$ amylase has been reported as a possible indicator for sympathetic nerve activity (15). In the results of this study, there was no significant difference in the salivary $\alpha$ amylase despite a decrease after massage. A potential reason for this is that individual differences affected the results as the salivary $\alpha$ amylase before massage was between 17.88 and 297.17, showing a big gap among the subjects. Thus, it was considered that the fact that the subjects were healthy women and their stress level was low affected the results. From this, salivary $\alpha$ amylase was not an effective indicator in this research.

From this, the significant increase in the pNN50 and the significant decrease in the heart rate meant the intervention of massage increased the autonomic nervous activity, increased the parasympathetic nerve activity and reduced the sympathetic nerve activity. This means the subjects were considered to be in a state of relaxation.

\section{Influence on emotion}

The following the influence on emotion, changes in anxiety, relaxation level, the willingness to communicate with other person, and the level of the sense of affinity are considered here.

State anxiety score shows temporal situational reactions while being in the state of anxiety. This score decreased significantly after massage, which was a result similar to those in a previous research (5). Furthermore, the trait anxiety score, which generally illustrates individual differences in comparably constant anxiety patterns, decreased significantly after massage. This was inferred that the temporal recognition at the moment had the possibility that it would correlate with a comparably stable recognition. Thus, it was suggested a possibility to reduce their anxiety, to provide regular hand massage to people with mental disorders who have anxiety.

There have been some previous studies which involve relaxation level measured by VAS as a measurement item (3-5). In such studies, the relaxation level was significantly increased after hand massage ; those were showed similar findings to this study. However, in the comparison with a study conducted by Matsushita et al., the variation ratio of the relaxation level was high in this study. The reason for this was considered as follows. We feared 
from the aspect of clinical knowledge that performing finger-pressure therapy (shiatsu massage) to patients with high anxiety or under pressure might be harmful and cause an adverse effect. We believed that it was more desirable to use physical contact based on soft touch to ease their anxiety. Based on this reason, the massage method employed in this study focused on the quality of the physical contact of soft touch by the hands of the performer to the subject, taking account of the subjects' feeling of "cherished". Hand massage with consideration on such matters may lead many recipients to feel relaxed.

In the field of oncology, there are reports which massage reduces the sense of isolation (16), however there have only been a small number of studies measuring a psychological distance as an indicator for the effects of hand massage.

In this study focused on the conversation need level and the sense of affinity as a manifestation of psychological distance, and measured its influence on emotion. As a result, the level of willingness to communicate with other person and the sense of affinity toward the massage-performer had a positive change of 70 percent. From this, it can be considered that a comfortable physical contact between a patient and a nursing profession, who are in a supported-supportive relationship, leads to an effect of shortening the gap in their psychological distance. Especially in the field of mental nursing where many patients have communication difficulty, it was suggested hand massage has a possibility to enhance the communication ability of such patients as it generates the willingness to communicate with other person and the sense of affinity. Also, hand massage can potentially be used as a way to construct a trust relationship with patients.

However, there were several limitations to this study : 1) This study was not randomized controlled. This should be done by accumulating results from experiments which go through the process of randomization. 2) As a progress in this field, it is necessary to accumulate the evidence that hand massage which is a type of physical contact based on soft touch can generate good physiological and psychological effects.

\section{CONCLUSION}

After performing hand massage which can be conducted conveniently regardless of location and can lead to mental relief, the subjects experienced a significant decrease in their heart rates and a significant increase in their pNN50s. This means their autonomic nervous activity increased, their parasympathetic nerve activity was improved and their sympathetic nerve activity was reduced. In addition, as there were a significant reduction in their anxiety, an increase in their relaxation level, and an increase in the willingness to communicate with other person and the level of the sense of affinity after performing hand massage.

\section{CONFLICT OF INTEREST}

None of the authors have any conflicts of interest to declare.

\section{ACKNOWLEDGMENTS}

The authors extend their sincere thanks to the participants who cooperated in the present study.

\section{REFERENCES}

1. Henderson $\mathrm{V}$ : Basic principles of nursing care. International Council of Nurses, Geneva, Switzerland, 1969

2. Kawahara Y, Morita M, Tanaka T, Okuda K, Hongo A, Tanaka A, Gomi K : Nurses' experiences of touch care : An analysis from the perspective of corporeality in the art of nursing. Japanese Journal of Nursing Art and Science 8(2) : 46-55, 2009 (in Japanese)

3. Sato $T$ : Effects of hand massage on the human autonomic nervous system and mood in healthy women. Yamanashi Nursing Journal 4(2) : 2532, 2006 (in Japanese)

4. Okamoto S, Ogawa T, Noda R, Emori Y : Relaxation effects of hand massage evaluated by salivary biomarker. Journal of Japan Society of Nursing Research 32(3) : 349, 2009 (in Japanese)

5. Matsushita M, Morishita T: A study on physiological alteration and psychological evaluation through purposeful touch. Journal of Mie Prefectural College of Nursing $7: 13-19,2003$ (in Japanese)

6. Ohkawa Y, Higashi S : Study of psysiological and psychological responses by hand massage 
in healthy women. The South Kyusyu Journal of Nursing 9(1) : 31-37, 2011 (in Japanese)

7. Ghazavi $Z$, Namnabati M, Faghihinia J, Mirbod M, Ghalriz P, Nekuie A, Fanian N : Effects of massage therapy of asthmatic children on the anxiety level of mothers. Iranian Journal of Nursing and Midwifery Research 15(3) : 130134, 2010

8. Chang MY, Wang SY, Chen $\mathrm{CH}$ : Effects of massage on Pain and Anxiety During : A Randomized Controlled Trial in Taiwan. J Adv Nurse 38(1) : 68-73, 2002

9. Field T, Morrow C, Valdeon C, Larson S, Kuhn $\mathrm{C}$, Schanberg S : Massage Reduces Anxiety in Child and Adolescent Psychiatric Patients. J Am Acad Child Adolesc Psychiatry 31(1) : 125-131, 1992

10. Degirmen N, Ozerdogan N, Sayiner D, Kosgeroglu N, Ayranci U : Effectiveness of foot and hand massage in postcesarean pain control in a group of Turkish pregnant women. Applied Nursing Research 23 : 153-158, 2010

11. Wang HL, Keck JF : Foot and hand massage as an intervention for postoperative pain. Pain Management Nursing 5(2) : 59-65, 2004

12. Spielberger $\mathrm{CD}:$ Anxiety as an emotional state. Anxiety current trends and theory. Academic press, New York, 1972

13. Groza p, Zamfir V, Lungu D : Postoperative salivary amylase changes in children. Rev Roum Physiol 8 : 307-312, 1971

14. Speirs RL, Herring J, Cooper WD, Hardy CC, Hind CR: The influence of sympathetic activity and isoprenaline on the secretion of amylase from the human parotid gland. Arch Oral Biol 19 : 747-752, 1974

15. Takai N, Yamaguchi M, Aragaki T, Eto K, Uchihashi K, Nishikawa Y : Effect of psychological stress on the salivary cortisol and amylase levels in healthy young adults. Arch Oral Biol 49 : 963-968, 2004

16. Macdonald $\mathrm{G}$ : The revival of hospital massage. Massage for the hospital patient and medically frail client. Lippincott Williams \& Wilkins, Philadelphia, 2005 